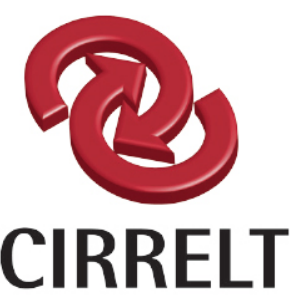

Centre interuniversitaire de recherche sur les réseaux d'entreprise, la logistique et le transport Interuniversity Research Centre

on Enterprise Networks, Logistics and Transportation

\title{
Population-Based Risk Equilibration for the Multi-Mode Hazmat Transport Network Design Problem
}

\author{
Pirmin Fontaine \\ Teodor Gabriel Crainic \\ Michel Gendreau \\ Stefan Minner
}

December 2018

CIRRELT-2018-56

Bureaux de Montréal : Université de Montréal Pavillon André-Aisenstadt C.P. 6128 , succursale Centre-ville Montréal (Québec)

Canada $\mathrm{H} 3 \mathrm{C} 3 \mathrm{~J} 7$

Téléphone : 514 343-7575

Télécopie : 514 343-7121

www.cirrelt.ca
Bureaux de Québec :

Université Laval

Pavillon Palasis-Prince

2325 , de la Terrasse, bureau 2642

Québec (Québec)

Canada G1V 0 A6

Téléphone : 418 656-2073

Télécopie : 418 656-2624

\begin{tabular}{|c|c|c|c|c|c|c|c|}
\hline 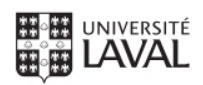 & McGill & 『Concordia & ÉTS & UQĀM & HEC MONTRËAL & $\begin{array}{l}\text { (Ф) POLYECHNIQUE } \\
\text { MONTRÉAL }\end{array}$ & $\begin{array}{l}\text { Université thy } \\
\text { de Montréal }\end{array}$ \\
\hline
\end{tabular}




\section{Population-Based Risk Equilibration for the Multi-Mode Hazmat Transport Network Design Problem ${ }^{\dagger}$}

\section{Pirmin Fontaine $^{1}$, Teodor Gabriel Crainic ${ }^{2,3,{ }^{*}, \text { Michel Gendreau}^{2,}{ }^{4}, \text { Stefan Minner }}{ }^{1}$}

${ }^{1}$ School of Management, Technical University of Munich, Arcisstraße 21, 80333 Munich, Germany

2 Interuniversity Research Centre on Enterprise Networks, Logistics and Transportation (CIRRELT)

3 Department of Management and Technology, Université du Québec à Montréal, P.O. Box 8888, Station Centre-Ville, Montréal, Canada H3C 3P8

${ }^{4}$ Department of Mathematics and Industrial Engineering, Polytechnique Montréal, P.O. Box 6079, Station Centre-Ville, Montréal, Canada H3C 3A7

Abstract. The shipment of hazardous materials is necessary for most countries and many of these products are flammable, explosive or radioactive. Despite high security standards, accidents still happen and the transportation of hazmat causes fear among the population who faces the risk of those accidents. Therefore, the society expects authorities to distribute the risk fairly. To support such a fair distribution, we propose a new population-based risk definition that evaluates the risk for the population in any given area with respect to its multimodal transportation network. Moreover, we propose different objective functions for equilibrating the risk and extend the bilevel Hazmat Transport Network Design Problem by considering several transportation modes. In this problem, the government wants to equilibrate the risk among the population centers by a restriction of links to the shipment of hazardous goods. When taking that decision, the government has to anticipate the reaction of the carriers who want to minimize the transportation costs. This bilevel problem is transformed into a single-level mixed-integer linear program and solved with Xpress. In the numerical results, we show that both objectives have a positive convex correlation and therefore a significant improvement in risk distribution can be achieved at the cost of just a small increase in total risk. The zones with a high risk will initially benefit from the risk redistribution. However, strong equilibrations just penalize zones with low risk. Moreover, compared to classical approaches in the literature, we achieve a better risk distribution among the population without increasing the total risk.

Keywords: Hazardous materials transportation, risk equilibration, network design.

Acknowledgements. While working on this project, the first author was also a postdoc at the School of Management, Université du Québec à Montréal and member of the Interuniversity Research Centre on Enterprise Networks, Logistics and Transportation (CIRRELT). The first author also gratefully acknowledges a fellowship of Deutscher Akademischer Austauschdienst (DAAD), which helped to start this work. While working on this project, the second and third authors were both also Adjunct Professors in the Department of Computer Science and Operations Research of the Université de Montréal. Partial funding for this project has been provided by the Natural Sciences and Engineering Research Council of Canada (NSERC), through its Discovery Grant program, and the Strategic Clusters program of the Fonds de recherche du Québec - Nature et technologie (FRQNT). The authors thank two anonymous referees whose comments have helped improve the paper.

${ }^{\dagger}$ Revised version of the CIRRELT-2016-63

Results and views expressed in this publication are the sole responsibility of the authors and do not necessarily reflect those of CIRRELT.

Les résultats et opinions contenus dans cette publication ne reflètent pas nécessairement la position du CIRRELT et n'engagent pas sa responsabilité.

* Corresponding author: TeodorGabriel.Crainic@cirrelt.ca

Dépôt légal - Bibliothèque et Archives nationales du Québec Bibliothèque et Archives Canada, 2018

(C) Fontaine, Crainic, Gendreau, Minner and CIRRELT, 2018 


\section{Introduction}

Hazardous material accidents can have tremendous consequences for the population. One of the worst accidents of this kind in recent transportation related history happened in July 2013 in Lac-Mégantic, Quebec, Canada. A driverless train with 72 tank cars of petroleum crude oil derailed in the city center, burning it to the ground, causing the death of at least 42 persons, and destroying at least 30 buildings and 115 businesses. It took almost 2 days to control the fire. Yet, the transport of hazardous materials is essential not only for industrial countries like Canada, Germany and the United States, but also for developing countries. The four most frequently shipped hazardous materials - with $80 \%$ of the transported volume in Canada - are crude petroleum, gasoline, fuel oils, and non-metallic minerals (Searag et al., 2015). According to the Bureau of Transportation Statistics and U.S. Census Bureau (2015), 2,580 million tons of hazardous materials were shipped throughout the United States in 2012. $59.4 \%$ of them were transported by truck, $4.3 \%$ by rail, $11 \%$ by water and $24.3 \%$ by pipeline in single mode transportation. Only $1 \%$ was shipped via intermodal transportation. In Canada, railways have a much higher relevance than in the USA. In 2012, 26.1 million tons were transported by rail and 107.4 million tons by truck. A different structure of the network in Germany, which, compared to North America, is very dense, is reflected in the share of transportation modes used: In 2010, 56 million tons were shipped by maritime transport, 48 million tons on inland waterways, 63 million tons by rail, and 140 million tons by trucks (Statistisches Bundesamt Wiesbaden, 2012).

The consideration of different transportation modes is thus essential to adequately represent the hazardous material transportation system, in particular for risk calculation whenever one contemplates regulating the industry. The review of the literature reveals, however, that the different streams of research investigate the transportation of hazardous materials from a single-mode point of view, considering it either on roads (e.g., Kara and Verter, 2004) or on rail (e.g., Verma et al., 2011, Hosseini and Verma, 2018). We fill this gap by integrating different transportation modes in the Hazmat Transport Network Design Problem (HTNDP).

This raises the double question of, first, how to compute risk and, second, how to equilibrate this risk over the considered population. With respect to the former question, risk in the literature is associated with the individual arcs of the network and the population zones arrayed along them (e.g., Kara and Verter, 2004). This definition neglects the fact that the risk to the population within any given area is influenced by the combined risk of all the transportation arcs, of all modes, crossing or bordering the area. We refer to such an area as a population center and introduce a new population-based risk definition to evaluate the risk in population centers.

Turning now to the latter question, one observes that, in the definitions proposed in the literature, either the total risk of the network is minimized (e.g., Kara and Verter, 2004) or the maximum arc risk is minimized for equilibration (e.g., Bianco et al., 2009). In most cases, these definitions do not yield a fair distribution of risk. Thus, e.g., even though the total risk over all arcs is minimized, a population center with a high number of arcs will face a higher risk than one with few arcs. Similarly, when one arc supports the transport of a high amount of hazardous material, the maximum risk in the network will be defined by that arc and the distribution of all other arcs and population centers becomes unimportant with respect to the maximum risk function. We therefore introduce and numerically compare several risk-equilibration functions, thus aiming at a fair distribution of risk among the populations. Our numerical results show that traditional risk measures are wanting with respect to the equilibration of risk for a given population, and that the new population-based measure, and the introduction of multimodality, yield a more effective model of risk and equity within a population. They also show that simply equilibrating risk may lead to a significant increase of the total risk in the network, and that all population centers may end up worse than before. Consequently, the selection of the appropriate measure is important, and we investigate the trade-off between risk equilibration and risk minimization, 
which shows a convex correlation between these two objectives. One can therefore achieve a much better distribution of risk among population centers with a small increase of the total risk in the network. To perform meaningful comparisons, we select and generalize, by introducing multiple transportation modes, the bilevel approach, a method well-known and well-represented in the literature. The resulting multimode multicommodity bilevel formulation is transformed into a mixed-integer linear program, and it is used in our numerical experiments to show the benefits of the new population-based risk definition over classical ones.

The contributions of this paper are: (1) A new population-based definition of risk and equity risk measures for hazardous material shipments; (2) an extension of the HTNDP to multimode transportation and risk equilibration; (3) a comparison of different risk equilibration measures; (4) insights into the trade-off between risk equilibration and risk minimization; (5) a comparison to existing models from the literature (single-mode and maximum arc risk equilibration).

This paper is structured as follows. The problem and notation are introduced, and the related literature is summarized, in Section 2. The new population-based risk definition is presented in Section 3. along with a number of risk-equilibration measures. Section 4 introduces the Multimode Hazmat Transport Network Design Problem and its transformation to a mixed-integer linear program. The results of the numerical study are presented and analyzed in Section 5 , before ending with the conclusion and an outlook on future research.

\section{Problem Definition and Related Literature}

According to Erkut et al. (2007), the literature on hazardous material transport can be classified into four categories: risk assessment, routing, combined facility location and routing, and network design. They give a summary on all of these topics. This paper is located in the area of risk assessment and network design, which, so far, has been the least investigated topic in this area.

The transportation network is represented by a graph $G=(N, A)$ with a set of nodes $N$ and a set of $\operatorname{arcs} A$. In a countrywide network, the nodes can be cities, facilities or important points in the network. In a city network, the level of detail needs to be much higher and the nodes represent junctions and entry and exit points of the city. Compared to the existing literature, we consider different transportation modes $m \in M$. Depending on the detail of the model, these modes are the classical modes train, road, rail, air, water and pipeline; however, we also define different vehicle types as transportation modes. To keep the notation simple, we neglect the fact that, in practice, not every arc can be used by every transportation mode. Each arc could further have a capacity limit for each mode. Especially pipelines have limitations on the possible amount of shipments. We, however, stay in line with the literature and neglect capacity restrictions.

$K$ is the set of commodities shipped through the network. Each commodity $k \in K$ is defined by an origin $o_{k} \in N$, a destination $d_{k} \in N$ and the transport volume $\phi_{k}$. The transportation costs for shipping one unit of commodity $k \in K$ on arc $(i, j) \in A$ with transportation mode $m \in M$ are $c_{i j}^{k m}$. Each commodity can be shipped partly via different transportation modes. However, we do not allow inter-modal transportation, as this is also not often the case in practice (e.g., Bureau of Transportation Statistics and U.S. Census Bureau, 2015). The probability of an incident on arc $(i, j) \in A$ with mode $m \in M$ is given by $\sigma_{i j}^{k m}$. Similar to the literature, it is assumed that there is no correlation between accidents and therefore the probability distributions are independent.

To equilibrate the risk among the population, we define a set of population centers $C$ with a pop- 
ulation $P_{c}$. In a global optimization setting, a population center represents a city; when the risk is equilibrated inside a city, these population centers represent districts or parts of the city. Such zones are well known in urban and regional planning (e.g., Crainic, T.G. and Florian, M., 2006, Crainic and Florian, 2008). In hazardous material routing, they were first introduced by Gopalan et al. (1990) for risk equity in a shortest-path problem. Further, Kang et al. (2014) and Taslimi et al. (2017) used zones in route planning and response team location for hazardous material transportation. These zones should be defined with respect to the surrounding axes of transport that may affect it.

Finally, $l_{i j}^{m k c}$ defines the influence of an accident on $\operatorname{arc}(i, j)$ of commodity $k$ using mode $m$ on the population $c$. This influence factor depends on the distance between the population center and the arc, as well as on the hazardous material type: The shorter the distance and the more dangerous the material is, the higher is the influence factor. The literature introduces different methods for calculating the influence of an accident on an arc: Batta and Chiu (1988) use a fixed bandwidth around the route segment, Erkut and Verter (1998) define a danger circle and Patel and Horowitz (1994) use a Gaussian plume model to define the impact of airborne hazmat accidents. We assume that these influence factors are given. Using this notation, we associate the risk with the population center. This is in contrast to the network design literature (e.g., Alp, 1995; Kara and Verter, 2004, Bianco et al., 2009), where risk calculation is associated with arcs. With $P_{i j}$ being the accumulated affected population in the area of $\operatorname{arc}(i, j)$, the classical literature defines the risk of an $\operatorname{arc}$ by $\sum_{k \in K} \sigma_{i j}^{k m} P_{i j} \phi_{k} x_{i j}^{k m}$ (Erkut and Verter. 1998). This definition is used in the models and solution methods for network design problems proposed in the literature.

To evaluate our new risk definition, we model the problem as a linear bilevel problem. In this wellstudied formulation, the leader represents the government or an authority. The leader can decide if the mode of a connection of the network is allowed for the transportation of hazardous materials or not and the decision is modeled by the binary decision variable $y_{i j}^{m}$. For simplification, we do not distinguish between specific hazardous material types, but the model could easily be extended to include this more realistic setting. The leader decision is subject to the follower optimization problem: The carriers minimize their transportation costs subject to demand satisfaction by deciding over the transportation percentage $x_{i j}^{k m}$ of commodity $k \in K$ shipped over arc $(i, j) \in A$ on transportation mode $m \in M$.

Kara and Verter (2004) reformulate the bilevel problem into a single-level formulation using the Karush-Kuhn-Tucker (KKT) conditions. In Verter and Kara (2008), a path-based formulation of the HTNDP is proposed. For each carrier, all possible paths are generated and ordered according to the carrier priority. Bianco et al. (2009) also transform the bilevel problem into a single-level formulation and show that their solutions might not be stable. If several follower solutions exist, the KKT conditions use an optimistic bilevel formulation that assumes that the carriers choose the path with the lowest risk among all shortest paths. In this case, the total risk can increase if a carrier chooses a path with a higher risk. They provide a method to evaluate the stability and present a heuristic that always finds stable solutions. Amaldi et al. (2011) propose a mixed-integer linear program for solving the HTNDP. This global optimization method further guarantees that stable solutions are found. Fontaine and Minner (2018) propose a Benders decomposition approach for solving larger instances of the classical HTNDP that only considers one transportation mode and risk minimization. Risk equilibration is not considered. While most models consider a network design problem, a different approach for a reduction of risk is the introduction of tolls for the transportation of hazardous materials (Marcotte et al. 2009, Esfandeh et al. 2016).

As linear bilevel problems are already $\mathcal{N} \mathcal{P}$-hard (Ben-Ayed and Blair, 1990), different heuristics are proposed as well: Erkut and Alp (2007) present a solution method that starts with a tree structured subset of the network and gradually adds new arcs. In contrast, Erkut and Gzara (2008) keep the bilevel formulation and propose a heuristic algorithm for solving the problem. The objective function of the follower problem is integrated into the objective function of the leader problem in a bi-objective bilevel 
model. Recently, Bianco et al. (2016) use a game-theoretic approach for regulating the transportation of hazardous materials via tolls. To calculate a Nash-equilibrium, they use a local search heuristic not only to minimize the total risk, but also to equilibrate the risk on the arcs by looking at the maximum risk on an arc. This approach is restricted to one hazmat type and the authors point out that an extension makes the problem much harder as the Nash game is no longer convex. Sun et al. (2015) include risk uncertainty into the network design problem and introduce a heuristic to find a robust solution. Bula et al. (2017) present a variable neighborhood search for the HazMat truckrouting problem, while Xie et al. (2012) combine location and routing decisions to minimize the risk in a multi-modal setting. Neither author addressed, however, network design or equilibration decisions.

Besides the solution method, the evaluation of risk for specific paths is also investigated. Alp (1995) minimizes the sum of the risk of all used arcs and Erkut and Ingolfsson (2000) propose more advanced objective functions like the mean-variance. In contrast to that, ReVelle et al. (1991) minimize the exposed population on a path and Saccomanno and Chan (1985) the incident probability on a path. Since all these measures assume independent risk probabilities, Kara et al. (2003) propose a method to evaluate the risk on a path accurately by including conditional risk distributions. Erkut and Verter (1998) show that the approximation error of the independent risk assumption is small. To include the fact that the population might not be risk-neutral and favor a higher probability of a low-consequences accident over a lower probability with high consequences, Abkowitz et al. (1992) introduce the perceived risk. The exposed population is exponentiated with a risk preference. If this risk preference is greater than 1 , a risk averse population is assumed, if it is 1, the population is risk neutral and if it is smaller than 1 , the population is risk prone. Bianco et al. (2009) and Bianco et al. (2016) equilibrate the risk over all arcs by minimizing the maximum risk on an arc. We will show that our approach can achieve a better distribution of the risk to a population by comparing it to these approaches. Moreover, Taslimi et al. (2017) propose a framework for equity in hazmat network design and hazmat response team location. The multi-modal effect is, however, not considered. A summary of different risk measures can also be found in Erkut and Ingolfsson (2005).

Even though risk equilibration is a fairly new topic to hazmat network design, it has been studied in the area of hazardous material routing. Gopalan et al. (1990) propose a shortest-path problem for routing trucks that minimizes the total risk and ensures risk equity between zones by a constraint. This constraint limits the risk deviation between the zones. However, the model ignores the fact that the carrier's main goal is cost minimization. Lindner-Dutton et al. (1991) extend this model and also include the sequencing of trucks in order to have a fair distribution in every period and not over the whole planning horizon. Carotenuto et al. (2007) define a mixed-interger linear program to find minimal and equitable risk routes for hazardous material shipments. This fair risk distribution is done by allocating it equally among the arcs. The problem is solved by a modified k-shortest path algorithm. For hazmat facility location, Romero et al. (2016) use the Gini coefficient to determine the location of storage facilities under the aspect of a fair risk distribution. Moreover, Sun et al. (2016) include cost equity for the carriers: Since network design decisions influence the costs of each carrier differently, the model equilibrates the cost increase of all carriers to avoid this unfair aspect.

\section{Population-based Risk Definition and Evaluation}

In this section, we first introduce the population-based risk definition. Then we define different possible risk equilibration measures and give an example for the differences between the classical risk definition and ours. 


\subsection{Risk Definition}

In contrast to the classical network design definition of risk on arcs, we define the risk for each population center $c \in C$. Following the classical definition, we assume that only one accident can happen at the same time on an arc. Therefore, the accidents on the different arcs through a center are independent and the expected risk is defined as follows:

$$
R_{c}(x):=P_{c} \sum_{m \in M} \sum_{k \in K} \sum_{(i, j) \in A} l_{i j}^{m k c} \sigma_{i j}^{k m} \phi_{k} x_{i j}^{k m}
$$

Thus, the risk of a population center is the sum of the transported volume on all arcs via all modes in the influence area of the center weighted with the accident risk, the potential influence factor, and the population size.

As long as the overall risk in the network is minimized, this risk definition is fully equivalent to the traditional risk definition, only the order of summation is changed. However, the differences can be huge for equilibrating the risk, i.e., by minimizing the maximum risk.

The network shown in Figure 1 gives an example of how the risk measures differ when we minimize the maximum risk. We assume two OD-pairs: 10 units from 1 to 4 via road and 10 units from 1 to 4 via rail. In this example, an optimal solution will always ship the 10 rail units via 2 , as no other solution

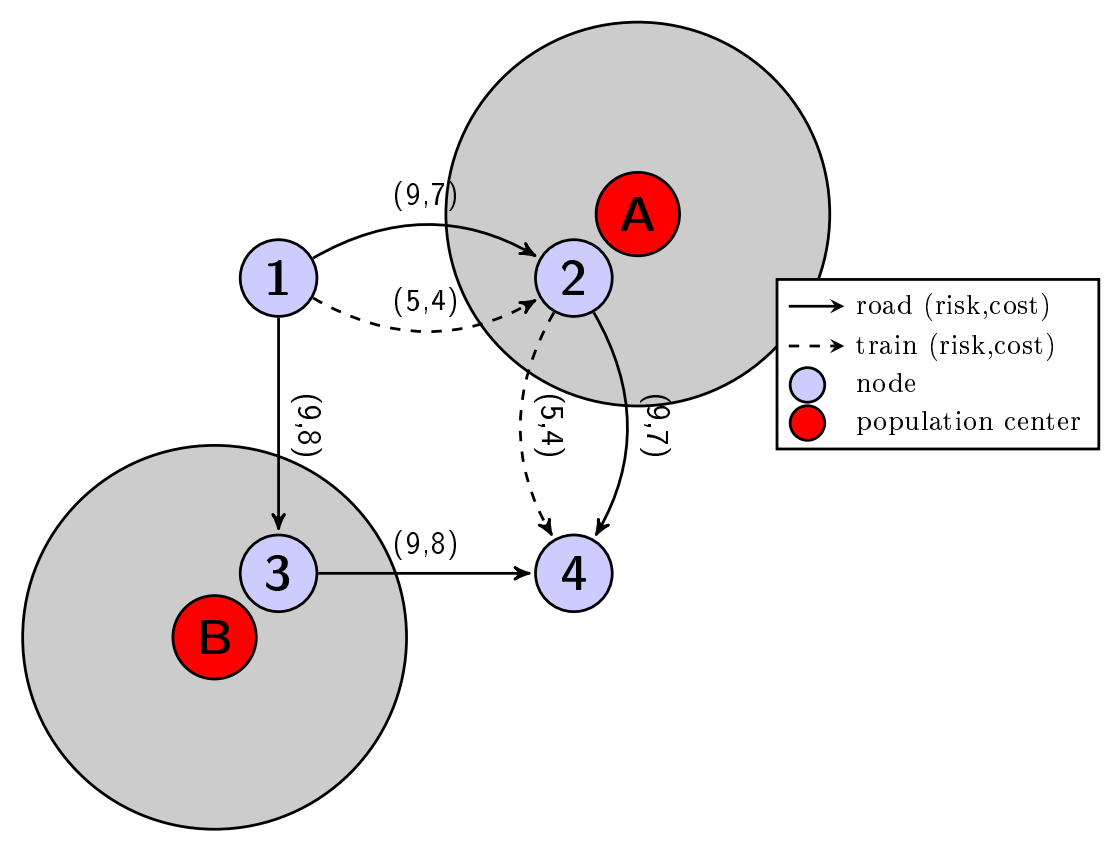

Figure 1: Example of the Population-based Risk Definition

exists. However, the road commodity has two options, shipping either via 2 or via 3 . If the maximum risk on each arc is minimized, both paths are the same from a risk perspective. Both will cause a total risk of 180 and no arc will be forbidden. The carrier will choose the cheapest path via 2 and population A will face a total risk of 280 and population B one of zero. If the maximum population risk is minimized, the government will close the road between 1 and 2 and the carrier will have to ship via 3 . The risk for population A would be 100 and for population B 180. In both solutions, the maximum risk on an arc is 90 and the classical risk in the network is 280 . The only difference is a fair distribution of the risk among the population. 


\subsection{Risk Equilibration Measures}

Erkut and Ingolfsson (2005) summarize different measures for the evaluation of risk on a path, Bianco et al. (2009) equilibrate the risk by minimizing the maximum risk on an arc and Marsh and Schilling (1994) review different equity measures in location theory. Following these risk evaluation ideas, we introduce several possible risk measures for the population-based risk definition of the whole network.

$$
\begin{aligned}
\sum_{c \in C} R_{c}(x) & \text { Traditional/Overall risk (Trad) } \\
\max _{c \in C} R_{c}(x) & \text { Maximum risk (Max) } \\
\frac{1}{|C|} \sum_{c \in C}\left|R_{c}(x)-\frac{1}{|C|} \sum_{c^{\prime} \in C} R_{c^{\prime}}(x)\right| & \text { Average deviation to mean (AdM) } \\
\max _{c \in C}\left|R_{c}(x)-\frac{1}{|C|} \sum_{c^{\prime} \in C} R_{c^{\prime}}(x)\right| & \text { Maximum deviation to mean (MdM) } \\
\frac{1}{|C|(|C|-1)} \sum_{c, c^{\prime} \in C \mid c<>c^{\prime}}\left|R_{c}(x)-R_{c^{\prime}}(x)\right| & \text { Average deviation among all (AdA) } \\
\max _{c, c^{\prime} \in C \mid c<>c^{\prime}}\left|R_{c}(x)-R_{c^{\prime}}(x)\right| & \text { Maximum deviation among all (MdA) }
\end{aligned}
$$

The traditional risk measure (2) sums the risk of all population centers and is equivalent to the arc definition of the risk. The maximum risk (3) minimizes the maximal risk in a population center. If each population center is defined by one arc, this definition is equivalent to the maximum arc risk definition by Bianco et al. (2009). The risk measures (5) - 7) are different deviation measures, all of which are zero when the risk is perfectly equilibrated and the risk (weighted with the population size) in every population center is the same. While the first two calculate the average and maximum deviation to the mean, the last two give the average difference between all population centers and the maximum difference between two population centers.

When only risk equilibration is considered without considering the level of the total risk of the network, some population centers may see their risk increase. To avoid this effect, we introduce a seventh risk measure with social bounds. Let $L$ be the set of social bounds. Then, each population center $c$ has the bounds $b_{c}^{l}$ for each $l \in L$. If the risk is higher than a bound, a penalty $p_{c}^{l}$ for all risk above this bound is added to the objective value. For a given number of $l$ bounds, this risk measure is defined as $\mathrm{Nl} l$ and calculated as follows:

$$
R_{c}(x)+\sum_{l \in L} p_{c}^{l} P_{c} \max \left\{0, \sum_{m \in M} \sum_{k \in K} \sum_{(i, j) \in A} l_{i j}^{m k c} \sigma_{i j}^{k m} x_{i j}^{k m}-b_{c}^{l}\right\} .
$$

This gives a piecewise linear increasing objective function. Such functions are also often used for equilibrating the user's travel time in traffic assignment problems (Sheffi, 1985). Moreover, this idea is similar to the idea of conditional value at risk and perceived risk with a risk-averse population. For example, Abkowitz et al. (1992) and Erkut and Ingolfsson (2000) use a non-linear function $f(x)=x^{\alpha}$ with $\alpha>1$ to take into account that accidents with high probability and low consequences are less undesirable than low probability-high consequence accidents. Compared to the first six risk measures, this measure combines risk equilibration and minimization. This is achieved through the traditional risk measure for the minimization, and through the social bounds for the equilibration. 


\section{Multi-Mode Hazmat Transport Network Design Problem}

In this section, we first introduce the bilevel formulation for the Multi-Mode Hazmat Transport Network Design Problem (mHTNDP) and explain how the general definition can be adapted to specific network types. Then we transform the model into a mixed-integer linear program.

\subsection{Bilevel Formulation}

Besides the already introduced decision variables $x_{i j}^{k m}$ and $y_{i j}^{m}$, let $z^{k m}$ be the percentage of commodity $k \in K$ shipped with mode $m$.

All introduced objective functions can be used in the leader problem, and all transformations shown in this section can be applied as well. Considering the maximum risk objective function, the leader problem can be defined as follows:

$$
\begin{aligned}
& \min r_{\max } \\
& \text { s.t. } P_{c} \sum_{m \in M} \sum_{k \in K} \sum_{(i, j) \in A} l_{i j}^{m k c} \sigma_{i j}^{k m} \phi_{k} x_{i j}^{k m} \leq r_{\max } \quad \forall c \in C \\
& r_{\max } \geq 0 \\
& y_{i j}^{m} \in\{0,1\}
\end{aligned}
$$

The follower problem is a multi-mode shortest path problem. The carriers decide how many percent $z^{k m}$ of commodity $k$ are shipped via transportation mode $m$. Equation (14 is the flow conservation constraint and constraint (15) ensures that the full demand is divided into the different transportation modes. Constraint (16) ensures that only arcs that are allowed by the leader can be used. In the objective function, the carriers' user-optimum - the overall transportation costs - are minimized.

$$
\begin{aligned}
& \min \sum_{k \in K} \sum_{m \in M} \sum_{(i, j) \in A} c_{i j}^{k m} x_{i j}^{k m} \\
& \text { s.t. } \quad \sum_{(i, j) \in A} x_{i j}^{k m}-\sum_{(j, l) \in A} x_{j l}^{k m}=\left\{\begin{array}{l}
0, \quad \text { if } j \neq o_{k}, d_{k} \\
-z^{k m}, \text { if } j=o_{k} \\
z^{k m}, \quad \text { if } j=d_{k}
\end{array} \quad \forall j \in N, k \in K, m \in M\right. \\
& \sum_{m \in M} z^{k m}=1 \quad \forall k \in K \\
& x_{i j}^{k m} \leq y_{i j}^{m} \\
& \forall(i, j) \in A, k \in K, m \in M \\
& x_{i j}^{k m} \geq 0 \\
& \forall(i, j) \in A, k \in K, m \in M \\
& z^{k m} \geq 0
\end{aligned}
$$

This model is a generalization of the classical HTNDP. Depending on the network and setting, several special cases are possible: By using only one transportation mode the follower problem is equivalent to the classical shortest path problem by Kara and Verter (2004). In an urban area setting, the only used transportation mode is the road with several vehicle types. Other transportation modes like rail or 
pipeline exist, but the decision might be taken in a global network design problem. However, for a fair equilibration, it is important to include the risk of these modes in the population center where it appears as constant.

By optimizing the risk distribution in a global setting like a province or a country, the modes can describe not only different vehicle types, but also rail, pipeline and water transport. Due to the transport via rail, pipeline or water, a capacity restriction might become necessary. However, with a capacity restriction, the follower problem becomes a multi-commodity transportation problem. Consequently, instead of a user-optimum, a system-optimum will be calculated, which is not considered in this paper. Consequently, the follower does not need to split up a shipment in the uncapacitated problem setting we consider in this paper. If different transportation modes have different costs (which is a reasonable assumption), the decision variable $z_{k m}$ will only take 0 and 1 as values. Thus, the reaction of the follower is either a different path on the same mode or a change of transportation mode.

\subsection{Transformation to a Mixed-Integer Linear Program}

To transform the linear bilevel problem into a non-linear mixed-integer program, we assume the partial cooperation assumption and the follower problem can be replaced by the Karush-Kuhn-Tucker conditions (Bard, 1998). The assumption of an optimistic bilevel problem can lead to unstable solutions. As introduced by Amaldi et al. (2011), an additional term can be added to the follower objective function to assume the pessimistic case. However, we used the optimistic case, since we also believe that carriers are not interested in choosing a high risk path and unique follower solutions are also possible by cost data perturbation.

$\min r_{\max }$

s.t.

$P_{c} \sum_{m \in M} \sum_{k \in K} \sum_{(i, j) \in A} l_{i j}^{m k c} \sigma_{i j}^{k m} \phi_{k} x_{i j}^{k m} \leq r_{\max } \quad \forall c \in C$

$\sum_{(i, j) \in A} x_{i j}^{k m}-\sum_{(j, l) \in A} x_{j l}^{k m}=\left\{\begin{array}{l}0, \quad \text { if } j \neq o_{k}, d_{k} \\ -z^{k m}, \quad \text { if } j=o_{k} \\ z^{k m}, \quad \text { if } j=d_{k}\end{array} \quad \forall j \in N, k \in K, m \in M\right.$

$\sum_{m \in M} z^{k m}=1 \quad \forall k \in K$

$x_{i j}^{k m} \leq y_{i j}^{m} \quad \forall(i, j) \in A, k \in K, m \in M$

$\sum_{k \in K} \sum_{m \in M} \sum_{(i, j) \in A} c_{i j}^{k m} x_{i j}^{k m} \leq \sum_{k \in K} v^{k}+\sum_{m \in M} \sum_{(i, j) \in A} \sum_{k \in K} t_{i j}^{k m} y_{i j}^{m}$

$u_{j}^{k m}-u_{i}^{k m}+t_{i j}^{k m} \leq c_{i j}^{k m}$

$\forall(i, j) \in A, k \in K, m \in M$

$u_{o_{k}}^{k m}-u_{d_{k}}^{k m}+v^{k} \leq 0$

$\forall k \in K, m \in M$

$v^{k} \in \mathbb{R}$

$\forall k \in K$

$u_{j}^{k m} \in \mathbb{R}$

$\forall j \in N, k \in K, m \in M$

$t_{i j}^{k m} \leq 0$

$\forall(i, j) \in A, k \in K, m \in M$

$x_{i j}^{k m} \geq 0$

$\forall(i, j) \in A, k \in K, m \in M$

$z^{k m} \geq 0$

$\forall k \in K, m \in M$ 


$$
\begin{aligned}
& r_{\max } \geq 0 \\
& y_{i j}^{m} \in\{0,1\}
\end{aligned}
$$

To replace the follower problem (13) - 18) with the optimality conditions, we define $u_{j}^{k m}, v^{k}$ and $t_{i j}^{k m}$ as the dual variables of the follower constraints (14) - 16). While (22) - (24) are the primal constraint, equation (26) is the dual constraint associated with the primal variable $x_{i j}^{k}$ and equation (27) is the dual constraint associated withthe primal variable $z^{k m}$. Equation 25 is the optimality condition that equals the primal and dual follower objectives.

As in Cao and Chen (2006), the optimality condition can be linearized by introducing the auxiliary variables $w_{i j}^{k m}$ and a $\operatorname{Big} \bar{M}$ and by replacing 25 with the following terms:

$$
\begin{array}{ll}
\sum_{k \in K} \sum_{m \in M} \sum_{(i, j) \in A} c_{i j}^{k m} x_{i j}^{k m} \leq \sum_{k \in K} v^{k}+\sum_{m \in M} \sum_{(i, j) \in A} \sum_{k \in K} w_{i j}^{k m} & \\
w_{i j}^{k m} \leq t_{i j}^{k m}+\hat{M}\left(1-y_{i j}^{m}\right) & \forall(i, j) \in A, m \in M, k \in K \\
w_{i j}^{k m} \geq t_{i j}^{k m} & \forall(i, j) \in A, m \in M, k \in K \\
w_{i j}^{k m} \geq-\hat{M} y_{i j}^{m} & \forall(i, j) \in A, m \in M, k \in K \\
w_{i j}^{k m} \leq 0 & \forall(i, j) \in A, m \in M, k \in K
\end{array}
$$

\section{$5 \quad$ Numerical Study}

In this section, we show give insights using the introduced measures of the previous section. We used Xpress 7.9 on an Intel Core i7 with 4 cores and 32GB RAM. In the results, we used the risk measure abbreviations of Section 3.2 ,

In the numerical study, we use two different networks as illustrative cases to show the benefits of our risk measure. In the first part, we use the Sioux Falls network from the literature (Bar-Gera, 2013) to show the convergence properties of the model. Then we show that it is important to analyze the trade-off between risk minimization and risk equilibration. Finally, we compare the model to risk formulations from the literature: a one-mode decision model and the maximum arc risk formulation. In the second part, we apply our model to a larger US-Canada instance (Orlowski et al., 2010) to verify the results of the small example.

\subsection{Sioux Falls Instances}

The Sioux Falls network consists of 24 nodes and 76 arcs. Each arc is defined by a length (in $\mathrm{km}$ ). Other necessary data is generated as follows: The network is divided into six population centers (the six gray areas in Figure 2). The population density of Sioux Falls is 814.4 inhabitants per square kilometer. The population size of each population zone is shown in the round brackets in Figure 2. The influence of an arc on a population center is set to 1 if the arc is inside the center, to 0 if not. If an arc is contained in more than one center, the influence is proportionally split into two parts (e.g., arc $(11,14)$ is $50 \%$ in the first and $50 \%$ in the second zone). 


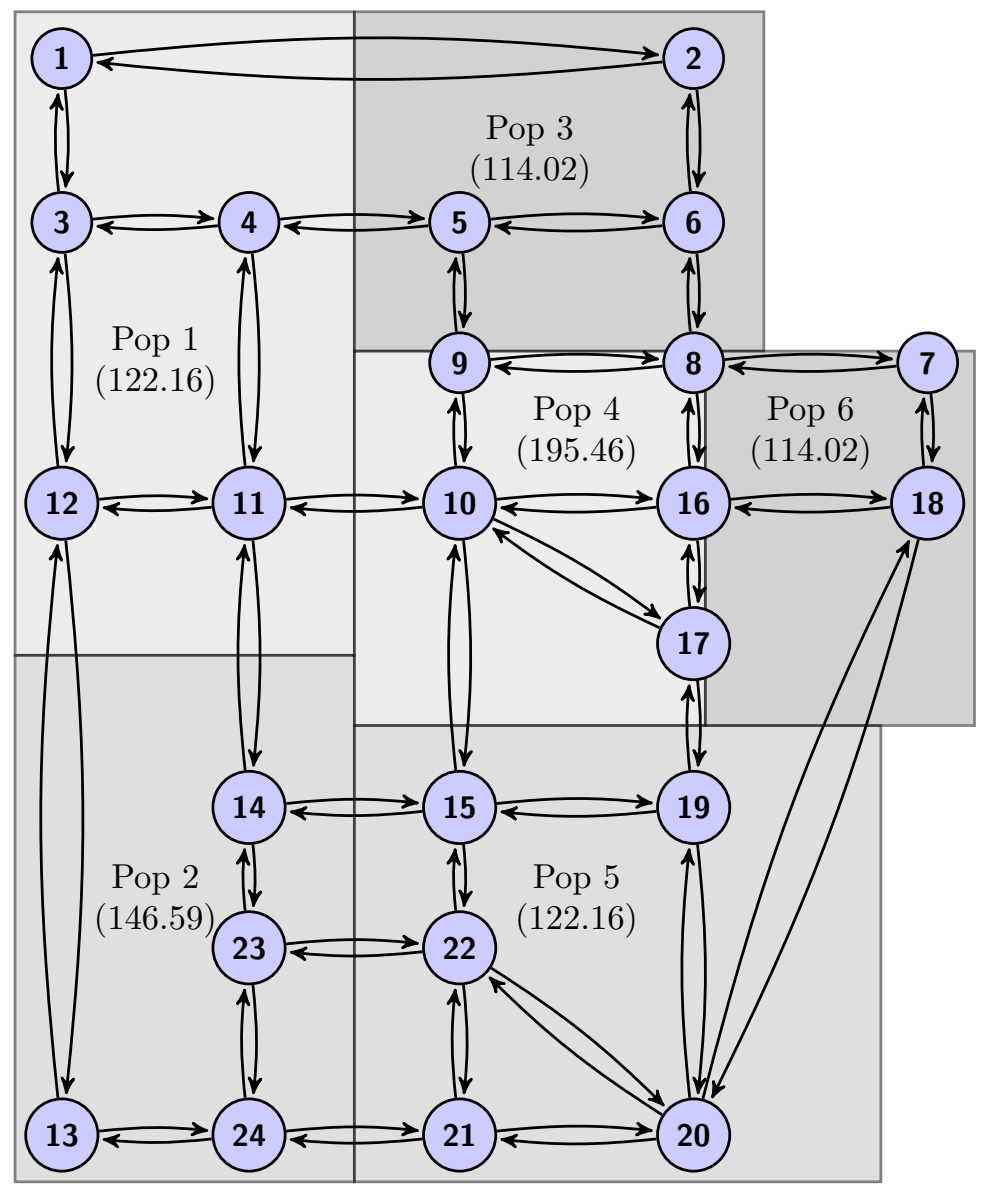

Figure 2: Sioux Falls Network with Population Centers and Population Density 
Two vehicle types with transportation costs of 1.1 per transported unit per $\mathrm{km}$ for the smaller vehicle and 0.9 per $\mathrm{km}$ for the larger vehicle are used. The accident probability of the larger vehicle is set $3 \%$ higher than the risk of the smaller one. The accident rate on an arc is generated randomly between $9.56 \times 10^{-9}$ and $1.08 \times 10^{-7}$ (Erkut and Gzara, 2008) and $\sigma_{i j}^{k m}$ is the product of the accident rate, the length of the arc and the factor for the vehicle type. We assume only one commodity type. Consequently, the accident rate on an arc is the same for all shipped commodities.

Four instances with different demand scenarios are generated. Nodes 1,2,13,20 are defined as the entry and exit nodes of the network. 43 commodities are shipped through the network (13 out-flows and 30 in-flows). The demand into the city (in-flows) is set between 100 and 1,000 and out of the city (out-flows) between 50 and 150. For each scenario, the origin, the destination, and the demand are generated randomly.

For the non-linear function (Equation (8)) with $l=7$ social bounds (NL7), we use a simple penalty function, which is defined as follows: The risk interval is divided into $l-1$ equidistant segments and from each beginning of an interval $p$, a further penalty of $p^{2}$ is added to the objective function.

In a first test, we evaluate the convergence of the equilibration function. Figure 3 shows that the lower bound stays zero and therefore the GAP cannot be calculated. This is the case for all measures that calculate the deviation: MdM, AdM, MdA, AdA. As mentioned in the definition of the measures, all population centers have the same weighted risk in an optimal equilibration. Thus, the optimal value is zero and the solver cannot calculate a lower bound other than zero. This effect, however, only appears for pure equilibration (Section 5.1.1). Most of the improvements happen within the first 20 minutes and

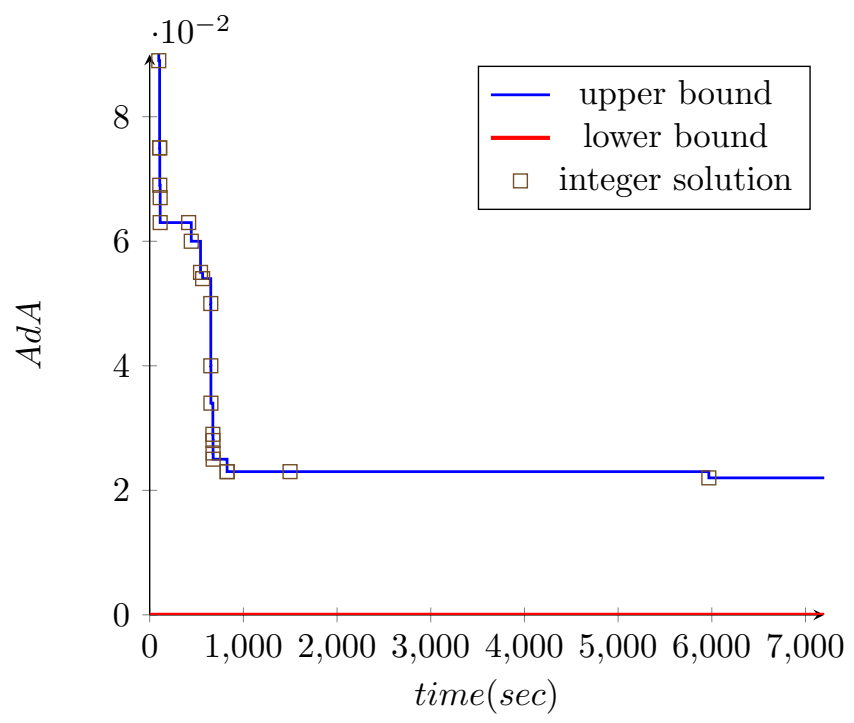

Figure 3: Convergence Analysis for AdA.

there are still some improvements during the first two hours. Therefore, the time limit of the numerical study was set to 7,200 seconds. Since the lower bound is larger than zero for all other objective functions and the trade-off calculations, the solver can use this lower bound to reduce the Branch-and-Cut tree and to calculate the GAP. Hence, the convergence was significantly better and the average gaps are below $1 \%$.

We first show the effect of risk equilibration and the differences between risk equilibration and total risk minimization and discuss possible approaches to combine them. Then, we compare the effect of 
using different transportation modes in one model before discussing the difference between our model and the equilibration idea found in the literature.

\subsubsection{Risk Equilibration}

Table 1 shows the results for the different objective functions for demand scenario 1 and the risk of all population centers is reported. The optimized objective function is shown in the first column. Moreover, the optimized risk measure is highlighted in bold.

Table 1: Risk Evalutation for Demand Scenario 1

\begin{tabular}{|c|c|c|c|c|c|c|c|c|c|c|c|c|}
\hline \multirow[t]{2}{*}{ Obj } & \multicolumn{6}{|c|}{ Risk measures } & \multicolumn{6}{|c|}{ Risk of population centers } \\
\hline & Trad & Max & AdM & $\mathrm{MdM}$ & AdA & MdA & Pop1 & Pop2 & Pop3 & Pop4 & Pop5 & Pop6 \\
\hline Trad & 1.574 & 0.525 & 0.155 & 0.262 & 0.207 & 0.419 & 0.525 & 0.179 & 0.464 & 0.105 & 0.169 & 0.132 \\
\hline Max & 2.144 & 0.370 & 0.018 & 0.055 & 0.023 & 0.067 & 0.370 & 0.367 & 0.366 & 0.303 & 0.370 & 0.370 \\
\hline AdM & 2.680 & 0.488 & 0.014 & 0.041 & 0.024 & 0.063 & 0.441 & 0.425 & 0.488 & 0.447 & 0.447 & 0.433 \\
\hline MdM & 2.251 & 0.441 & 0.042 & 0.066 & 0.065 & 0.128 & 0.314 & 0.371 & 0.441 & 0.314 & 0.421 & 0.390 \\
\hline AdA & 3.194 & 0.541 & 0.004 & 0.012 & 0.007 & 0.020 & 0.520 & 0.534 & 0.541 & 0.532 & 0.533 & 0.532 \\
\hline $\mathrm{MdA}$ & 2.482 & 0.444 & 0.028 & 0.042 & 0.040 & 0.072 & 0.414 & 0.372 & 0.444 & 0.443 & 0.436 & 0.372 \\
\hline NL7 & 1.574 & 0.525 & 0.155 & 0.262 & 0.207 & 0.419 & 0.525 & 0.179 & 0.464 & 0.105 & 0.169 & 0.132 \\
\hline
\end{tabular}

The results show that just minimizing the deviation or the maximum leads to an extreme increase in the overall risk in the network. The results are quite obvious as an equilibrium is only possible on a high level. It shows that, for an equal distribution of risk, almost every population center comes out worse and the total risk increases by more than $100 \%$. Only the non-linear function NL7, which does not try to equalize all populations, distributes the risk better without a dramatic risk increase. These effects are similar for the other 3 instances. The maximum risk is only effective if there is no population center with a very high risk and the equilibration measures AdM, MdM, AdA, MdM perform very similar But since AdA shows the best performance in terms of equilibration, we will use this measure for equilibrating risk in the following analysis.

\subsubsection{Trade-off between Risk Equilibration and Risk Minimization}

Since the pure risk equilibration increases the total risk significantly, we analyze the trade-off between minimizing the total risk in the network and equilibrating the risk. In the literature (e.g., Gopalan et al. 1990, Lindner-Dutton et al., 1991), this is achieved by adding the level of equilibration as a constraint into the model. This approach is not applicable in bilevel programming, as this constraint would be a so-called coupling constraint (e.g., Dempe, 2002) and would then make the KKT-transformation invalid. Therefore, we used a bi-objective function that combines the AdA equilibration measure with the overall risk function Trad, which is weighted with $\alpha \in\{0.1,0.15,0.2,0.25,0.3,0.35,0.4,0.45,0.5\}$. As mentioned earlier, the optimality gaps are significantly smaller in this study. The bi-objective function has an average gap of less than $1 \%$ over all instances.

Table 2 shows the results for the 4 randomly generated demand scenarios (DS). Besides the total risk, the equilibration measure, and the risk of each population, we further show the routing effect of the new bi-objective solution compared to the traditional risk measure. We report if the follower adjusted the 
Table 2: Trade-off between Trad and AdA for 4 Demand Scenarios

\begin{tabular}{|c|c|c|c|c|c|c|c|c|c|c|c|}
\hline \multirow[t]{2}{*}{ DS } & \multirow[t]{2}{*}{ Objective function } & \multicolumn{2}{|c|}{ Risk measure } & \multicolumn{6}{|c|}{ Risk of population centers } & \multicolumn{2}{|c|}{ Effect } \\
\hline & & Trad & $\mathrm{AdA}$ & Pop 1 & Pop 2 & Pop 3 & Pop 4 & Pop 5 & Pop 6 & Route & Mode \\
\hline \multirow[t]{10}{*}{1} & Trad & 1.574 & 0.207 & 0.525 & 0.179 & 0.464 & 0.105 & 0.169 & 0.132 & & \\
\hline & $\mathrm{AdA}+0.50$ Trad & 1.637 & 0.136 & 0.381 & 0.272 & 0.430 & 0.163 & 0.227 & 0.164 & $19 \%$ & $5 \%$ \\
\hline & $\mathrm{AdA}+0.45$ Trad & 1.643 & 0.133 & 0.402 & 0.261 & 0.430 & 0.180 & 0.190 & 0.179 & $21 \%$ & $14 \%$ \\
\hline & $\mathrm{AdA}+0.40$ Trad & 1.643 & 0.133 & 0.402 & 0.261 & 0.430 & 0.180 & 0.190 & 0.179 & $21 \%$ & $14 \%$ \\
\hline & $\mathrm{AdA}+0.35$ Trad & 1.660 & 0.126 & 0.357 & 0.283 & 0.442 & 0.186 & 0.210 & 0.181 & $21 \%$ & $14 \%$ \\
\hline & $\mathrm{AdA}+0.30$ Trad & 1.726 & 0.104 & 0.348 & 0.279 & 0.423 & 0.202 & 0.274 & 0.200 & $30 \%$ & $21 \%$ \\
\hline & $\mathrm{AdA}+0.25$ Trad & 1.862 & 0.068 & 0.346 & 0.284 & 0.423 & 0.269 & 0.272 & 0.269 & $49 \%$ & $19 \%$ \\
\hline & $\mathrm{AdA}+0.20$ Trad & 2.024 & 0.031 & 0.329 & 0.328 & 0.406 & 0.321 & 0.320 & 0.320 & $30 \%$ & $37 \%$ \\
\hline & $\mathrm{AdA}+0.15$ Trad & 2.074 & 0.029 & 0.351 & 0.337 & 0.400 & 0.327 & 0.331 & 0.328 & $23 \%$ & $58 \%$ \\
\hline & $\mathrm{AdA}+0.10$ Trad & 2.179 & 0.015 & 0.370 & 0.362 & 0.378 & 0.357 & 0.370 & 0.341 & $37 \%$ & $26 \%$ \\
\hline \multirow[t]{10}{*}{2} & Trad & 1.388 & 0.177 & 0.393 & 0.175 & 0.448 & 0.164 & 0.134 & 0.074 & & \\
\hline & $\mathrm{AdA}+0.50$ Trad & 1.425 & 0.151 & 0.383 & 0.208 & 0.417 & 0.129 & 0.161 & 0.127 & $12 \%$ & $12 \%$ \\
\hline & $\mathrm{AdA}+0.45$ Trad & 1.425 & 0.151 & 0.383 & 0.208 & 0.417 & 0.129 & 0.161 & 0.127 & $12 \%$ & $12 \%$ \\
\hline & $\mathrm{AdA}+0.40$ Trad & 1.480 & 0.127 & 0.372 & 0.243 & 0.383 & 0.147 & 0.187 & 0.148 & $14 \%$ & $9 \%$ \\
\hline & $\mathrm{AdA}+0.35$ Trad & 1.480 & 0.127 & 0.372 & 0.243 & 0.383 & 0.147 & 0.187 & 0.148 & $14 \%$ & $14 \%$ \\
\hline & $\mathrm{AdA}+0.30$ Trad & 1.494 & 0.122 & 0.374 & 0.247 & 0.375 & 0.152 & 0.194 & 0.152 & $28 \%$ & $23 \%$ \\
\hline & $\mathrm{AdA}+0.25$ Trad & 1.584 & 0.098 & 0.368 & 0.263 & 0.368 & 0.193 & 0.199 & 0.193 & $14 \%$ & $51 \%$ \\
\hline & $\mathrm{AdA}+0.20$ Trad & 1.983 & 0.014 & 0.340 & 0.335 & 0.347 & 0.320 & 0.322 & 0.320 & $37 \%$ & $19 \%$ \\
\hline & $\mathrm{AdA}+0.15$ Trad & 2.063 & 0.002 & 0.343 & 0.343 & 0.346 & 0.344 & 0.342 & 0.345 & $42 \%$ & $35 \%$ \\
\hline & $\mathrm{AdA}+0.10$ Trad & 2.043 & 0.003 & 0.341 & 0.340 & 0.346 & 0.340 & 0.337 & 0.339 & $58 \%$ & $14 \%$ \\
\hline \multirow[t]{10}{*}{3} & Trad & 1.399 & 0.159 & 0.377 & 0.199 & 0.412 & 0.185 & 0.154 & 0.072 & & \\
\hline & $\mathrm{AdA}+0.50$ Trad & 1.447 & 0.125 & 0.358 & 0.250 & 0.370 & 0.145 & 0.189 & 0.136 & $9 \%$ & $23 \%$ \\
\hline & $\mathrm{AdA}+0.45$ Trad & 1.447 & 0.125 & 0.358 & 0.250 & 0.370 & 0.145 & 0.189 & 0.136 & $9 \%$ & $23 \%$ \\
\hline & $\mathrm{AdA}+0.40$ Trad & 1.540 & 0.084 & 0.323 & 0.306 & 0.323 & 0.180 & 0.237 & 0.170 & $14 \%$ & $30 \%$ \\
\hline & $\mathrm{AdA}+0.35$ Trad & 1.546 & 0.082 & 0.322 & 0.308 & 0.323 & 0.178 & 0.238 & 0.177 & $12 \%$ & $33 \%$ \\
\hline & $\mathrm{AdA}+0.30$ Trad & 1.546 & 0.082 & 0.322 & 0.308 & 0.323 & 0.178 & 0.238 & 0.177 & $12 \%$ & $33 \%$ \\
\hline & $\mathrm{AdA}+0.25$ Trad & 1.654 & 0.051 & 0.319 & 0.303 & 0.325 & 0.235 & 0.237 & 0.235 & $28 \%$ & $28 \%$ \\
\hline & $\mathrm{AdA}+0.20$ Trad & 1.764 & 0.027 & 0.314 & 0.304 & 0.325 & 0.275 & 0.273 & 0.274 & $40 \%$ & $40 \%$ \\
\hline & $\mathrm{AdA}+0.15$ Trad & 1.843 & 0.011 & 0.310 & 0.302 & 0.327 & 0.303 & 0.301 & 0.301 & $14 \%$ & $30 \%$ \\
\hline & $\mathrm{AdA}+0.10$ Trad & 1.875 & 0.007 & 0.322 & 0.308 & 0.318 & 0.307 & 0.309 & 0.310 & $21 \%$ & $47 \%$ \\
\hline \multirow[t]{10}{*}{4} & Trad & 1.335 & 0.165 & 0.347 & 0.217 & 0.419 & 0.099 & 0.171 & 0.081 & & \\
\hline & $\mathrm{AdA}+0.50$ Trad & 1.402 & 0.115 & 0.310 & 0.280 & 0.339 & 0.129 & 0.233 & 0.112 & $12 \%$ & $16 \%$ \\
\hline & $\mathrm{AdA}+0.45$ Trad & 1.432 & 0.100 & 0.301 & 0.306 & 0.312 & 0.125 & 0.258 & 0.131 & $14 \%$ & $26 \%$ \\
\hline & $\mathrm{AdA}+0.40$ Trad & 1.443 & 0.096 & 0.301 & 0.306 & 0.309 & 0.136 & 0.258 & 0.133 & $23 \%$ & $12 \%$ \\
\hline & $\mathrm{AdA}+0.35$ Trad & 1.443 & 0.095 & 0.301 & 0.305 & 0.309 & 0.136 & 0.259 & 0.133 & $23 \%$ & $12 \%$ \\
\hline & $\mathrm{AdA}+0.30$ Trad & 1.458 & 0.091 & 0.301 & 0.304 & 0.310 & 0.143 & 0.257 & 0.143 & $21 \%$ & $23 \%$ \\
\hline & $\mathrm{AdA}+0.25$ Trad & 1.641 & 0.042 & 0.301 & 0.304 & 0.310 & 0.237 & 0.255 & 0.234 & $47 \%$ & $23 \%$ \\
\hline & $\mathrm{AdA}+0.20$ Trad & 1.768 & 0.015 & 0.306 & 0.304 & 0.308 & 0.289 & 0.284 & 0.278 & $30 \%$ & $30 \%$ \\
\hline & $\mathrm{AdA}+0.15$ Trad & 1.794 & 0.010 & 0.298 & 0.310 & 0.308 & 0.294 & 0.294 & 0.289 & $33 \%$ & $21 \%$ \\
\hline & $\mathrm{AdA}+0.10$ Trad & 1.799 & 0.009 & 0.303 & 0.308 & 0.307 & 0.298 & 0.290 & 0.293 & $33 \%$ & $23 \%$ \\
\hline
\end{tabular}


shipment of a commodity by rerouting using the same mode ("Route") or by changing the transportation mode ("Mode"). The numbers show the percentage of all commodities that change their shipment.

The minimal risk solutions are 1.574 (DS 1), 1.388 (DS 2), 1.399 (DS 3) and 1.335 (DS 4) and shown in the first line of each scenario. One can see that the risk is better distributed among the population centers without increasing the risk as much as in the pure equilibration measures. Also, as expected, a higher weight on the risk minimization (Trad) leads, to a lower risk with a worse equilibration.

The distribution is already much better for an increase of $5-15 \%$ of the total risk. However, to distribute the risk as fairly as possible, an increase of more than $35 \%$ is necessary. Moreover, the equilibration of the first $\alpha$ steps is mainly achieved by a significant reduction of the risk in the population centers with the highest risk and a shift to low risk population centers. But especially in the last steps, the equilibration is achieved by increasing the risk in low risk population centers without reducing the risk in high risk population centers. For example in demand scenario 1, the risk of population center 1 drops from 0.525 to 0.381 in the first step $(\alpha=0.5 \%)$. Population center 3 improves in the first step from 0.464 to 0.430 . Even though the equilibration improves further for $\alpha \leq 0.5$, this is mostly due to a risk increase in population centers $2,4,5$ and 6 .

A similar effect can be seen in the other scenarios. DS2 shows a significant improvements of population centers 1 and 3 until $\alpha=0.4$. After that, the equilibration is again mostly achieved by increasing the risk in other population centers.

In the last two columns, one can see that the redistribution of risk is achieved through both rerouting and mode change. As reported earlier, a too strong equilibration is not desirable since only population centers with low risk are penalized. In these cases, the effect on rerouting and transportation mode change are strong. As soon as the traditional risk measure is weighted with 0.3 for scenario 1 and 2 (with 0.25 for scenario 3 and 4 ) or less, more than $50 \%$ of the commodities are affected. In the other cases, this effect is smaller. However, both options are used. This emphasizes that several transportation modes should be considered when trying to fairly distribute the risk in a network.

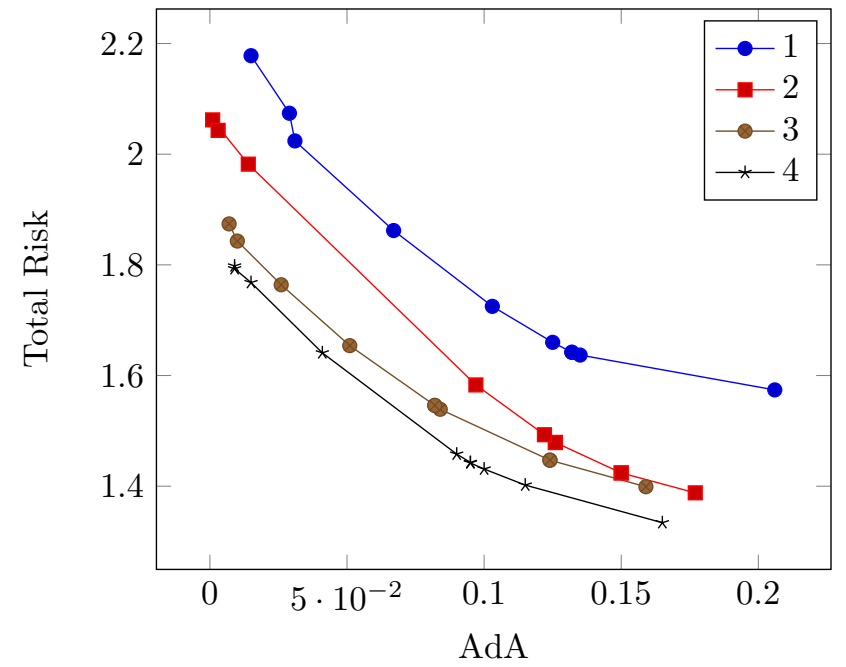

Figure 4: Trade-off between Trad and AdA for 4 Demand Scenarios

Figure 4 shows the Pareto-optimal curves of the trade-off between an equilibrated network and a network with a low total risk. The results indicate a convex substitution relation on the efficient frontier 
between both objective functions, which is consistent with our previous findings: With a small increase of total risk, the risk can be much better equilibrated. However, there comes a point from which on the price of the total risk in the network for further equilibration is very high.

Besides the bi-objective approach, the non-linear objective function of the previous section also shows similar effects and can be a good alternative, especially when risk is perceived differently in different population centers.

\subsubsection{Comparison to One-Mode Decision Model}

In this subsection, we show the necessity of using a multi-mode decision model by comparing our model to classical single mode models. In the multi-mode decision model, the mode is part of the decision process. Therefore, we split the demands according to the modal split of our multi-mode network design model. The resulting demand patterns are used for solving the hazmat network design problem for mode 1 and 2 separately and for evaluating the sum of both ("sum" in Table 3). The multi-mode result is compared with the sum of the single mode models. Moreover, the network design of the two single mode models is used in the multi-mode model to see the reaction of the followers to the single mode decisions ("reaction"). For all models, we used the non-linear objective function with 7 approximation points (NL7) as the non-linear function combines risk equilibration and risk minimization in one function. The detailed results are shown in Table 3.

Table 3: Comparison of the multi-mode model with single-level decisions

\begin{tabular}{|c|c|c|c|c|c|c|c|c|c|}
\hline \multirow[t]{2}{*}{ DS } & \multirow[t]{2}{*}{ Model } & \multicolumn{2}{|c|}{ Risk measure } & \multicolumn{6}{|c|}{ Risk of population centers } \\
\hline & & Trad & AdA & Pop 1 & Pop 2 & Pop 3 & Pop 4 & Pop 5 & Pop 6 \\
\hline \multirow[t]{3}{*}{1} & ulti mode & 1.696 & 0.119 & 0.357 & 0.286 & 0.413 & 0.147 & 0.284 & 0.20 \\
\hline & $\mathrm{su}$ & 1.687 & 0.127 & 381 & 0.298 & 0.402 & 130 & .267 & 0.209 \\
\hline & reaction & 1.681 & 0.130 & 378 & 0.294 & 0.410 & 128 & 4 & 0.207 \\
\hline \multirow[t]{3}{*}{2} & multi mode & 1.524 & 0.11 & 0.356 & 0.288 & 0.365 & 147 & 0.211 & 0.1 \\
\hline & sum & 1.522 & 0.1 & 356 & 0.289 & 0.365 & 0.147 & 0.208 & 0.157 \\
\hline & reaction & 1.522 & 0.1 & 0.356 & 0.289 & 0.365 & 0.147 & 0.208 & 0.157 \\
\hline \multirow[t]{3}{*}{3} & multi mode & 1.538 & 0.08 & 0.326 & 0.293 & 0.329 & 0.208 & 0.239 & 0.142 \\
\hline & sum & 1.547 & 0.099 & 0.362 & 0.267 & 0.329 & 0.215 & 0.233 & 0.140 \\
\hline & reaction & 1.460 & 0.142 & 0.386 & 0.210 & 0.396 & 0.188 & 0.183 & 0.097 \\
\hline \multirow[t]{3}{*}{4} & multi mode & 1.419 & 0.110 & 0.303 & 0.286 & 0.336 & 0.143 & 0.240 & 0.112 \\
\hline & & 1.423 & 01 & $0:$ & 0.329 & 0.303 & 0.090 & 0.262 & 0.131 \\
\hline & reaction & 1.424 & 0.118 & 0.308 & 0.329 & 0.303 & 0.090 & 0.262 & 0.131 \\
\hline
\end{tabular}

In scenario 2, there is no difference between the three models. In the other three scenarios, the distribution gets worse. In scenario 1 , the total risk is reduced by $5 \%$, but the equilibration is worse by $60 \%$. Population centers 1 and 3, which are the ones with the highest risk, increase their risk. This shift towards risk minimization is mostly caused by the reaction of the followers if they are again allowed to change their mode. In scenario 4 , the single mode models result in an even higher total risk and a worse equilibration. This worse equilibration is due to the fact that two equilibrated modes do not need to be equilibrated in the same way as when several modes are considered.

This shows that the consideration of different modes in the models is important. The effect of solving the different modes separately depends on the instance and can lead to different risk distributions than with single modes. 


\subsubsection{Comparison to Maximum Arc Risk Equilibration}

To equilibrate risk, the literature so far proposed to minimize the maximum arc risk (Bianco et al. 2009). In Table 4, we compare the solution of a maximum arc risk model to solutions of the Pareto curve of the previous section for the four different demand scenarios.

Table 4: Comparison to Maximum Arc Risk Model

\begin{tabular}{|c|c|c|c|c|c|c|c|c|c|}
\hline \multirow[t]{2}{*}{ DS } & \multirow{2}{*}{$\begin{array}{l}\text { Objective } \\
\text { function }\end{array}$} & \multicolumn{2}{|c|}{ Risk measure } & \multicolumn{6}{|c|}{ Risk of population centers } \\
\hline & & Trad & AdA & Pop 1 & Pop 2 & Pop 3 & Pop 4 & Pop 5 & Pop 6 \\
\hline \multirow[t]{3}{*}{1} & $\max \operatorname{arc}$ & 1.890 & 0.217 & 0.591 & 0.211 & 0.534 & 0.210 & 0.209 & 0.135 \\
\hline & 0.25 Trad & 1.862 & 0.068 & 0.346 & 0.284 & 0.423 & 0.269 & 0.272 & 0.269 \\
\hline & Trad & 1.574 & 0.207 & 0.525 & 0.179 & 0.464 & 0.105 & 0.169 & 0.132 \\
\hline \multirow[t]{4}{*}{2} & $\max \operatorname{arc}$ & 1.789 & 0.173 & 0.458 & 0.264 & 0.503 & 0.209 & 0.211 & 0.144 \\
\hline & AdA +0.25 Trad & 1.584 & 0.098 & 0.368 & 0.263 & 0.368 & 0.193 & 0.199 & 0.193 \\
\hline & AdA +0.20 Trad & 1.983 & 0.014 & 0.340 & 0.335 & 0.347 & 0.320 & 0.322 & 0.320 \\
\hline & Trad & 1.388 & 0.177 & 0.393 & 0.175 & 0.448 & 0.164 & 0.134 & 0.074 \\
\hline \multirow[t]{3}{*}{3} & $\max \operatorname{arc}$ & 1.975 & 0.170 & 0.447 & 0.254 & 0.508 & 0.378 & 0.247 & 0.142 \\
\hline & AdA +0.10 Trad & 1.875 & 0.007 & 0.322 & 0.308 & 0.318 & 0.307 & 0.309 & 0.310 \\
\hline & Trad & 1.399 & 0.159 & 0.377 & 0.199 & 0.412 & 0.185 & 0.154 & 0.072 \\
\hline \multirow[t]{3}{*}{4} & $\max \operatorname{arc}$ & 1.760 & 0.176 & 0.450 & 0.350 & 0.426 & 0.178 & 0.271 & 0.086 \\
\hline & AdA +0.20 Trad & 1.768 & 0.015 & 0.306 & 0.304 & 0.308 & 0.289 & 0.284 & 0.278 \\
\hline & Trad & 1.335 & 0.165 & 0.347 & 0.217 & 0.419 & 0.099 & 0.171 & 0.081 \\
\hline
\end{tabular}

The results show for all demand scenarios that there exists a solution with a similar total risk in the network but a better distribution among the population centers and a solution with a similar distribution within the population centers but a significantly smaller total risk. The use of a maximum arc risk increased the total risk by more than $35 \%$ without distributing the risk better. In all scenarios, every population center has a higher risk than in the risk minimal solution. However, compared to the equilibration measures for population centers, this does not lead to a better distribution of risk. The risk distribution remains more or less the same as in the minimal risk solution. Therefore, a similar risk distribution is always possible with the minimal overall risk solution.

This shows that the maximum arc formulation leads to a very unbalanced risk distribution among the population.

\section{$5.2 \quad$ US-Canada Instance}

As a second instance, we use a network of the United States and Canada (Orlowski et al., 2010), which is shown in Figure 5. The network consists of 39 nodes (cities in Canada and the United States) and 122 directed arcs. The transportation costs are given, and the length of an arc are calculated by using the Euclidean distance between the two nodes. Each city defines one population center. The population of each center is the population of the city and the influence factor is set to 0.5 for the origin and the destination cities of an arc. The accident rates on an arc and $\sigma_{i j}^{k m}$ are generated in the same way as for the Sioux Falls network. Two vehicle types are used, the second vehicle costs $5 \%$ more while the first vehicle's accident probability is $3 \%$ higher. A set of 100 commodities with randomly chosen origin and destination pairs is generated. The demand is defined randomly between 300 and 600 . 


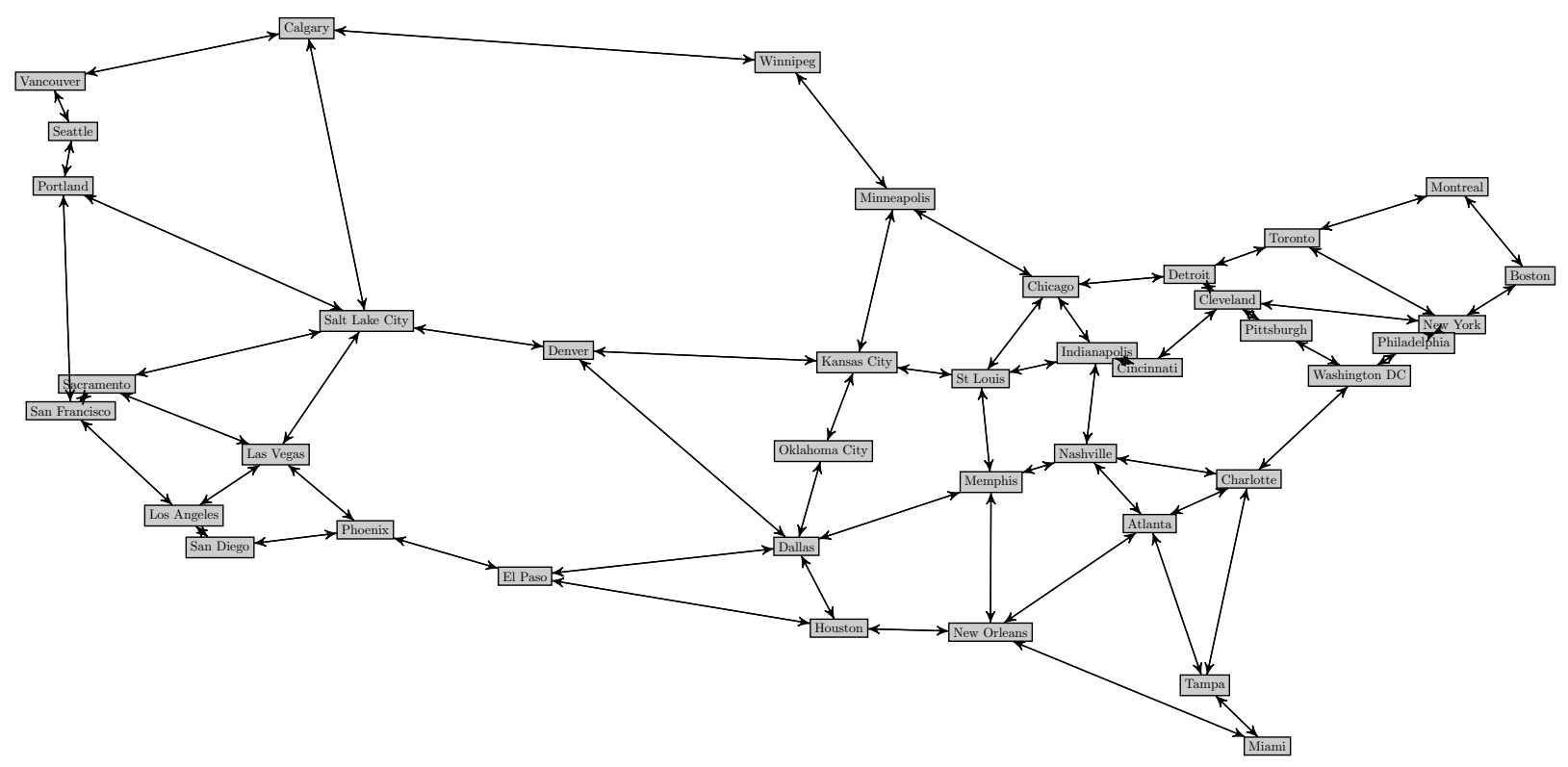

Figure 5: United States and Canada Network

We use the AdA as equilibration measure and compare the unregulated network with the risk minimal solution, the maximum arc risk solution and several bi-objective functions. The time limit is set to 10 hours and the average gap is below $5 \%$ (excluding the maximum arc risk).

The computational results in Table 5 show that the unregulated network has a very high overall risk, a very poor distribution and some cities can have a very high risk. Using the classical approach, the total risk and the distribution can be improved by almost $50 \%$. The maximum risk for a population is reduced from 9.075 to 4.146 , which is more than $50 \%$. Using the maximum arc risk formulation from the literature, we find that the maximum risk for a population is similar to that of the Trad risk function. However, the total risk in the network is significantly higher and the distribution is worse. For the bi-objective function, one can see again that a small increase in total risk leads to a significantly better distribution. An increase of the total risk by $7 \%$ improves the distribution by $20 \%$ and the maximum risk of a population center is reduced by over $40 \%$. However, further risk increases only lead to small improvements in the distribution. In fact, for the last case, even though the distribution improves, the maximum risk of a population increases to 2.744 .

Table 5: Risk Comparison for the US-Canada Instance

\begin{tabular}{rrrr}
\hline Objective & Trad & AdA & max Pop \\
\hline free network & 60.694 & 1.684 & 9.075 \\
max arc & 48.429 & 1.195 & 4.144 \\
Trad & 31.368 & 0.802 & 4.146 \\
AdA +0.03 Trad & 33.654 & 0.634 & 2.417 \\
AdA +0.02 Trad & 36.237 & 0.625 & 2.428 \\
AdA +0.015 Trad & 37.750 & 0.613 & 2.417 \\
AdA +0.01 Trad & 39.798 & 0.597 & 2.744 \\
\hline
\end{tabular}

Moreover, we analyze the changes within the population centers. The risk of each city for the different 
risk measures are shown in Table 6. Comparing the Trad solution with the AdA+0.03Trad solution, we find that 8 out of 13 cities among the first third of those with the highest risk reduce their risk by $24 \%$ on average while of the cities in the last third, 12 suffer an increase of $120 \%$ on average. In total numbers, this equals a reduction of 4.338 in the first third and an increase by 2.507 .

This effect, however, changes when comparing AdA+0.03Trad with AdA+0.02Trad. Only 7 cities in the first third reduce their risk by a total of 0.880 while the risk in 10 cities in the last third increases by 3.121. From $A d A+0.02 T r a d$ to $A d A+0.015 T r a d$, the risk of 9 out of 13 cities of the first third reduces again, while the risk of 8 out of 13 of the last third increases. However, the reduction is again only 0.778, whereas the increase is 3.507. This again supports the assumption of the aggregated numbers: At the beginning, the cities with a high risk can benefit from the risk redistribution, but too strong equilibration just penalizes cities with a low risk.

\section{Conclusions}

We introduced a new population-based risk definition and extended the HTNDP to a multi-mode problem in order to address the problem of risk equity. In the numerical study, we showed the superiority of the new definition over the arc risk definition and that it is necessary to consider multiple modes in the model. We also showed that the pure equilibration of risk increases the total risk significantly and that decision makers have to find a trade-off between equilibration and risk minimization. However, because of the convex correlation between these two measures, a small increase in the total risk can lead to a much better equilibration. As the problem is still very difficult to solve, enhancements for solving it should be considered in further research. Moreover, a differentiation between various hazardous material classes seems to be interesting. Using different classes could lead to an even better distributions of risk. Since bilevel problems in general do not lead to robust solutions, the investigation of robustness and a method to generate robust solutions should also be considered in future research. Even though intermodal shipments represent a small share of hazardous material transportation, considering them could give further insights on whether they could be used for significantly decreasing the risk in hazardous material transportation. This consideration, however, is not without difficulties, since it involves accounting for the risk incurred when changing from one mode to another.

\section{Acknowledgements}

While working on this project, the first author was also a postdoc at the School of Management, Universityé du Québec à Montréal and member of the Interuniversity Research Centre on Enterprise Networks, Logistics and Transportation (CIRRELT). The first author also gratefully acknowledges a fellowship of Deutscher Akademischer Austauschdienst (DAAD), which helped to start this work. While working on this project, the second and third authors were both also Adjunct Professors in the Department of Computer Science and Operations Research of the Université de Montréal. Partial funding for this project has been provided by the Natural Sciences and Engineering Council of Canada (NSERC), through its Discovery Grant program, and the Strategic Clusters program of the Fonds québécois de la recherche sur la nature et les technologies. The authors thank two anonymous referees whose comments have helped improve the paper. 
Table 6: Risk of Population Center for the US-Canada Instance

\begin{tabular}{|c|c|c|c|c|c|c|c|}
\hline & free network & $\max \operatorname{arc}$ & Trad & $\begin{array}{c}\text { AdA + } \\
0.03 \text { Trad }\end{array}$ & $\begin{array}{c}\text { AdA }+ \\
0.02 \text { Trad }\end{array}$ & $\begin{array}{r}\text { AdA }+ \\
0.015 \text { Trad }\end{array}$ & $\begin{array}{c}\text { AdA }+ \\
0.01 \text { Trad }\end{array}$ \\
\hline Atlanta & 0.996 & 0.829 & 0.618 & 0.697 & 0.596 & 0.811 & 0.626 \\
\hline Boston & 0.236 & 0.206 & 0.205 & 0.204 & 0.204 & 0.204 & 0.249 \\
\hline Calgary & 2.845 & 1.610 & 0.513 & 1.738 & 1.279 & 1.589 & 1.392 \\
\hline Charlotte & 1.392 & 0.920 & 0.429 & 0.509 & 0.729 & 0.673 & 0.852 \\
\hline Chicago & 7.182 & 3.090 & 1.054 & 2.065 & 1.926 & 2.006 & 1.865 \\
\hline Cincinnati & 0.267 & 0.254 & 0.254 & 0.285 & 0.411 & 0.444 & 0.442 \\
\hline Cleveland & 0.561 & 0.545 & 0.425 & 0.499 & 0.729 & 0.784 & 0.794 \\
\hline Dallas & 4.037 & 3.187 & 0.932 & 1.143 & 0.992 & 0.885 & 1.664 \\
\hline Denver & 4.115 & 4.144 & 4.146 & 2.086 & 2.428 & 2.221 & 2.123 \\
\hline Detroit & 1.267 & 0.996 & 0.501 & 0.499 & 0.547 & 0.592 & 0.626 \\
\hline El Paso & 1.262 & 0.722 & 0.331 & 0.766 & 0.752 & 0.849 & 1.025 \\
\hline Houston & 1.533 & 1.399 & 0.558 & 0.868 & 1.862 & 1.600 & 1.153 \\
\hline Indianapolis & 1.723 & 1.336 & 1.361 & 1.520 & 1.472 & 1.291 & 1.346 \\
\hline Kansas City & 2.717 & 2.911 & 3.335 & 2.274 & 2.263 & 2.258 & 2.292 \\
\hline Las Vegas & 0.597 & 0.625 & 0.379 & 0.547 & 0.633 & 0.778 & 0.819 \\
\hline Los Angeles & 1.167 & 2.058 & 0.630 & 0.505 & 0.607 & 1.099 & 0.882 \\
\hline Memphis & 2.455 & 2.178 & 1.167 & 0.822 & 0.902 & 0.960 & 1.087 \\
\hline Miami & 0.143 & 0.104 & 0.076 & 0.432 & 0.637 & 0.515 & 0.708 \\
\hline Minneapolis & 0.723 & 0.498 & 0.274 & 0.550 & 0.491 & 0.673 & 0.555 \\
\hline Montreal & 0.690 & 1.393 & 0.819 & 0.814 & 0.814 & 0.814 & 1.129 \\
\hline Nashville & 2.276 & 1.710 & 1.047 & 0.976 & 0.729 & 1.070 & 0.981 \\
\hline New Orleans & 0.597 & 0.618 & 0.482 & 0.575 & 0.827 & 0.834 & 1.049 \\
\hline New York & 9.075 & 3.798 & 2.414 & 2.417 & 2.425 & 2.417 & 2.021 \\
\hline Oklahoma City & 0.094 & 0.096 & 0.152 & 0.216 & 0.205 & 0.172 & 0.264 \\
\hline Philadelphia & 0.658 & 0.634 & 0.650 & 0.650 & 0.654 & 0.716 & 0.794 \\
\hline Phoenix & 1.742 & 0.594 & 0.445 & 0.763 & 1.022 & 0.885 & 1.344 \\
\hline Pittsburgh & 0.172 & 0.280 & 0.355 & 0.401 & 0.415 & 0.398 & 0.414 \\
\hline Portland & 0.878 & 1.074 & 0.660 & 0.613 & 0.858 & 0.781 & 0.913 \\
\hline Sacramento & 0.418 & 0.915 & 0.363 & 0.509 & 0.587 & 0.784 & 0.961 \\
\hline Salt Lake City & 0.889 & 1.266 & 0.871 & 0.494 & 0.637 & 0.461 & 0.451 \\
\hline San Diego & 0.455 & 0.117 & 0.189 & 0.275 & 0.452 & 0.217 & 0.766 \\
\hline San Francisco & 0.290 & 0.219 & 0.160 & 0.408 & 0.359 & 0.942 & 0.739 \\
\hline Seattle & 0.242 & 0.380 & 0.238 & 0.487 & 0.589 & 0.778 & 0.739 \\
\hline St. Louis & 1.205 & 1.410 & 1.470 & 1.062 & 1.081 & 0.992 & 1.081 \\
\hline Tampa & 0.320 & 0.207 & 0.169 & 0.491 & 0.547 & 0.388 & 0.445 \\
\hline Toronto & 2.403 & 3.871 & 2.341 & 2.331 & 2.331 & 2.331 & 2.744 \\
\hline Vancouver & 0.620 & 0.229 & 0.184 & 0.432 & 0.587 & 0.781 & 0.739 \\
\hline Washington DC & 0.679 & 0.831 & 0.782 & 0.801 & 0.849 & 0.784 & 0.810 \\
\hline Winnipeg & 1.771 & 1.175 & 0.388 & 0.930 & 0.812 & 0.975 & 0.913 \\
\hline
\end{tabular}




\section{References}

M. D. Abkowitz, M. Lepofsky, and P. Cheng. Selecting criteria for designating hazardous materials highway routes. Transportation Research Record, 1333:30-35, 1992.

E. Alp. Risk-based transportation planning practice: Overall methodology and a case example. Infor, 33(1):4, 1995.

E. Amaldi, M. Bruglieri, and B. Fortz. On the hazmat transport network design problem. In J. Pahl, T. Reiners, and S. Voß, editors, Network Optimization Lecture Notes in Computer Science, volume 6701, pages 327-338. Springer Berlin Heidelberg, 2011.

H. Bar-Gera. Transportation network test problems, 2013. URL http://www.bgu.ac.il/ bargera/ $\operatorname{tntp} /$.

J. F. Bard. Practical Bilevel Optimization: Algorithms and Applications. Springer, 1998.

R. Batta and S. S. Chiu. Optimal obnoxious paths on a network: transportation of hazardous materials. Operations Research, 36(1):84-92, 1988.

O. Ben-Ayed and C. E. Blair. Computational difficulties of bilevel linear programming. Operations Research, 38(3):556-560, 1990.

L. Bianco, M. Caramia, and S. Giordani. A bilevel flow model for hazmat transportation network design. Transportation Research Part C: Emerging Technologies, 17(2):175-196, 2009.

L. Bianco, M. Caramia, S. Giordani, and V. Piccialli. A game-theoretic approach for regulating hazmat transportation. Transportation Science, 50(2):424-438, 2016.

G. A. Bula, C. Prodhon, F. A. Gonzalez, H. M. Afsar, and N. Velasco. Variable neighborhood search to solve the vehicle routing problem for hazardous materials transportation. Journal of Hazardous Materials, 324:472 - 480, 2017. ISSN 0304-3894.

Bureau of Transportation Statistics and U.S. Census Bureau. Transportation-commodity flow survey. Technical report, 2015.

D. Cao and M. Chen. Capacitated plant selection in a decentralized manufacturing environment: A bilevel optimization approach. European Journal of Operational Research, 169(1):97-110, 2006.

P. Carotenuto, S. Giordani, and S. Ricciardelli. Finding minimum and equitable risk routes for hazmat shipments. Computers $\& 3$ Operations Research, 34(5):1304-1327, 2007.

T.G. Crainic and M. Florian. National Planning Models and Instruments. INFOR, 46(4):81-90, 2008.

Crainic, T.G. and Florian, M. Modèles d'optimisation pour la planification des systèmes de transport. In Paschos, V., editor, Optimisation combinatoire : applications, pages 117-152. Hermès Lavoisier, Paris, 2006.

S. Dempe. Foundations of bilevel programming. Springer Science \& Business Media, 2002.

E. Erkut and O. Alp. Designing a road network for hazardous materials shipments. Computers $\mathcal{E}$ Operations Research, 34(5):1389-1405, 2007.

E. Erkut and F. Gzara. Solving the hazmat transport network design problem. Computers 83 Operations Research, 35(7):2234-2247, 2008.

E. Erkut and A. Ingolfsson. Catastrophe avoidance models for hazardous materials route planning. Transportation Science, 34(2):165-179, 2000. 
E. Erkut and A. Ingolfsson. Transport risk models for hazardous materials: revisited. Operations Research Letters, 33(1):81-89, 2005.

E. Erkut and V. Verter. Modeling of transport risk for hazardous materials. Operations research, 46(5): 625-642, 1998.

E. Erkut, S. A. Tjandra, and V. Verter. Chapter 9 hazardous materials transportation. In C. Barnhart and G. Laporte, editors, Transportation, volume 14 of Handbooks in Operations Research and Management Science, pages 539 - 621. Elsevier, 2007.

T. Esfandeh, C. Kwon, and R. Batta. Regulating hazardous materials transportation by dual toll pricing. Transportation Research Part B: Methodological, 83:20 - 35, 2016. ISSN 0191-2615.

P. Fontaine and S. Minner. Benders decomposition for the hazmat transport network design problem. European Journal of Operational Research, 267(3):996 - 1002, 2018. ISSN 0377-2217.

R. Gopalan, K. S. Kolluri, R. Batta, and M. H. Karwan. Modeling equity of risk in the transportation of hazardous materials. Operations Research, 38(6):961-973, 1990.

S. D. Hosseini and M. Verma. Conditional value-at-risk (cvar) methodology to optimal train configuration and routing of rail hazmat shipments. Transportation Research Part B: Methodological, 110:79 - 103, 2018. ISSN 0191-2615.

Y. Kang, R. Batta, and C. Kwon. Generalized route planning model for hazardous material transportation with var and equity considerations. Computers \& Operations Research, 43:237-247, 2014.

B. Y. Kara and V. Verter. Designing a road network for hazardous materials transportation. Transportation Science, 38(2):188-196, 2004.

B. Y. Kara, E. Erkut, and V. Verter. Accurate calculation of hazardous materials transport risks. Operations Research Letters, 31(4):285-292, 2003.

L. Lindner-Dutton, R. Batta, and M. H. Karwan. Equitable sequencing of a given set of hazardous materials shipments. Transportation Science, 25(2):124-137, 1991.

P. Marcotte, A. Mercier, G. Savard, and V. Verter. Toll policies for mitigating hazardous materials transport risk. Transportation Science, 43(2):228-243, 2009.

M. T. Marsh and D. A. Schilling. Equity measurement in facility location analysis: A review and framework. European Journal of Operational Research, 74(1):1-17, 1994.

S. Orlowski, R. Wessäly, M. Pióro, and A. Tomaszewski. Sndlib 1.0 survivable network design library. Networks, 55(3):276-286, 2010.

M. H. Patel and A. J. Horowitz. Optimal routing of hazardous materials considering risk of spill. Transportation Research Part A: Policy and Practice, 28(2):119-132, 1994.

C. ReVelle, J. Cohon, and D. Shobrys. Simultaneous siting and routing in the disposal of hazardous wastes. Transportation Science, 25(2):138-145, 1991.

N. Romero, L. K. Nozick, and N. Xu. Hazmat facility location and routing analysis with explicit consideration of equity using the gini coefficient. Transportation research part E: logistics and transportation review, 89:165-181, 2016.

F. F. Saccomanno and A.-W. Chan. Economic evaluation of routing strategies for hazardous road shipments. Transportation Research Record, 1020, 1985.

S. Searag, G. Maloney, and L. McKeown. Trucking dangerous goods in canada, 2004 to 2012. Technical report, Statistics Canada, 2015. 
Y. Sheffi. Urban Transportation Networks: Equilibrium Analysis with Mathematical Programming Methods. Prentice-Hall, Inc., 1985.

Statistisches Bundesamt Wiesbaden. Verkehr Gefahrguttransporte. Fachserie 8, Reihe 1.4, 2012.

L. Sun, M. H. Karwan, and C. Kwon. Robust hazmat network design problems considering risk uncertainty. Transportation Science, 2015.

L. Sun, M. H. Karwan, and C. Kwon. Implications of cost equity consideration in hazmat network design. Transportation Research Record: Journal of the Transportation Research Board, (2567):67-77, 2016.

M. Taslimi, R. Batta, and C. Kwon. A comprehensive modeling framework for hazmat network design, hazmat response team location, and equity of risk. Computers $\&$ Operations Research, 79:119-130, 2017.

M. Verma, V. Verter, and M. Gendreau. A tactical planning model for railroad transportation of dangerous goods. Transportation Science, 45(2):163-174, 2011.

V. Verter and B. Y. Kara. A path-based approach for hazmat transport network design. Management Science, 54(1):29-40, 2008.

Y. Xie, W. Lu, W. Wang, and L. Quadrifoglio. A multimodal location and routing model for hazardous materials transportation. Journal of Hazardous Materials, 227-228:135 - 141, 2012. ISSN 0304-3894. 